\title{
UNION RESULTS FOR THIN SETS
}

\author{
by KATHRYN E. HARE $\dagger$
}

(Received 8 November, 1988; revised 24 April, 1989)

0. Introduction. Let $G$ be a compact abelian group and let $\Gamma$ be its (discrete) dual group. Denote by $M(G)$ the space of complex regular Borel measures on $G$.

Let $E$ be a subset of $\Gamma$. Then:

(i) $E$ is called a Rajchman set if, for all $\mu \in M(G), \underset{\gamma \in \Gamma \backslash E}{\lim \sup } \hat{\mu}(\gamma)=0$ implies $\lim \sup \hat{\mu}(\gamma)=0$ [15];

(ii) $E$ is called a set of continuity if given $\varepsilon>0$ there exists $\delta>0$ such that if $\mu \in M(G),\|\mu\| \leq 1$ and $\limsup _{\gamma \in \Gamma \backslash E}|\hat{\mu}(\gamma)|<\delta$, then $\limsup _{\gamma \in E}|\hat{\mu}(\gamma)|<\varepsilon[6]$; and

(iii) $E$ is called a parallelepiped of dimension $N$ if $|E|=2^{N}$ and there are two-element sets $\left\{\chi_{i}, \psi_{i}\right\} \subset \Gamma, i=1, \ldots, N$ so that $E=\prod_{i=1}^{N}\left\{\chi_{i}, \psi_{i}\right\}$ [5]. (The multiplication indicated here is the group operation.)

The classical result of Rajchman [16] to the effect that $\mathbb{Z}^{+}$and $\mathbb{Z}^{-}$are Rajchman sets inspired the first definition. Subsequently, de Leeuw and Katznelson [2] proved that $\mathbb{Z}^{+}$ and $\mathbb{Z}^{-}$are sets of continuity. Clearly any set of continuity is a Rajchman set, but the converse is not true [5]. As $\mathbb{Z}^{+} \cup \mathbb{Z}^{-}$is neither a Rajchman set nor a set of continuity it is natural to ask under what conditions the union of two Rajchman sets (sets of continuity) is another Rajchman set (set of continuity). In Section 1 we establish the following results.

THEOREM A. The union of a Rajchman set with a set which does not contain parallelepipeds of arbitrarily large dimension is again a Rajchman set.

THEOREM B. The union of a proportional set of continuity or a strong set of continuity with a set not containing parallelepipeds of arbitrarily large dimension is a set of continuity. (See Section 1 for definitions.)

As examples of thin sets which do not contain arbitrarily large parallelepipeds include $\Lambda(p)$ sets [9], Theorem A extends Pigno's results in [15] to the effect that the union of a Rajchman set and a Sidon set is a Rajchman set, as is the union of $\mathbb{Z}^{-}$and certain $\Lambda(p)$ sets. Theorem B extends a result of Fournier and Pigno [5] that the union of $\mathbb{Z}^{+}$and a set not containing arbitrarily large parallelepipeds is a set of continuity, as $\mathbb{Z}^{+}$is a proportional set of continuity.

In Section 2 we show that the absence of parallelepipeds of arbitrarily large dimension does not characterize $\Lambda(p)$ sets for $p>2$. This extends a similar result in [9] which requires $p>8 / 3$.

1. Union results. Our proofs of Theorems A and B are basically combinatorial arguments which depend on the arithmetic characterizations of Rajchman sets and sets of continuity discovered by Host and Parreau $[10,11]$. We need the following definition and notation to describe these characterizations.

$\dagger$ Research supported in part by the NSERC and the University of Waterloo.

Glasgow Math. J. 32 (1990) 241-254. 
A set $\left\{\theta_{j}\right\}_{j \geq 1} \subseteq \Gamma$ is called a dissociate set if for each positive integer $N$, the relation $\prod_{j=1}^{N} \theta_{j}^{\varepsilon_{j}}=1$ with $\varepsilon_{j}=0, \pm 1, \pm 2$ implies $\theta_{j}^{\varepsilon_{j}}=1$ for all $j=1, \ldots, N$.

Notation. For a dissociate set $\theta=\left\{\theta_{j}\right\}_{j \geq 1}$, let

and

$$
\Omega(\theta)=\left\{\prod_{j=1}^{N} \theta_{j}^{\varepsilon_{j}}: \varepsilon_{j}=0, \pm 1, N \in \mathbb{N}\right\}
$$

$$
\Omega_{n}(\theta)=\left\{\prod_{j=1}^{N} \theta_{j}^{\varepsilon_{j}}: \varepsilon_{j}=0, \pm 1, \sum_{j=1}^{N}\left|\varepsilon_{j}\right| \leq n, N \in \mathbb{N}\right\} .
$$

The characterization of Rajchman sets and sets of continuity are:

Theorem I [11, Theorem 1] (see also [10, Theorem 1]). A set $E \subseteq \Gamma$ is a Rajchman set if and only if $E$ does not contain $\alpha \Omega(\theta)$ for any $\alpha \in \Gamma$ and infinite dissociate set $\theta \subseteq \Gamma$.

TheOREM II [10, Theorem 3]. A set $E \subseteq \Gamma$ is a set of continuity if and only if for some positive integer $n, E$ does not contain $\alpha \Omega_{n}(\theta)$ for any $\alpha \in \Gamma$ and infinite dissociate set $\theta \subseteq \Gamma$.

Theorem II suggests ways one could modify the notion of a set of continuity. Call $E$ a strong set of continuity if there exists a positive integer $n$ so that $E$ does not contain $\alpha \Omega\left(\left\{\theta_{1}, \ldots, \theta_{n}\right\}\right)$ for any $\alpha \in \Gamma$ and dissociate set $\left\{\theta_{i}\right\}_{i=1}^{n}$. Call $E$ a proportional set of continuity if there is a constant $C<1$ (called the proportionality constant) and a positive integer $n_{0}$ such that for $\alpha \in \Gamma$, an infinite dissociate set $\left\{\theta_{j}\right\}_{j \geq 1}$ and $n \geqslant n_{0}$,

$$
\liminf _{N \rightarrow \infty} \frac{\left|E \cap \alpha \Omega_{n}\left(\left\{\theta_{1}, \ldots, \theta_{N}\right\}\right)\right|}{\left|\alpha \Omega_{n}\left(\left\{\theta_{1}, \ldots, \theta_{N}\right\}\right)\right|} \leq C .
$$

(Here $|\cdot|$ denotes cardinality.)

Obviously sets which do not contain parallelepipeds of arbitrarily large dimension are strong sets of continuity. We will show, and use the fact later, that they are also proportional sets of continuity.

It is unknown if there are sets of continuity which are not proportional sets of continuity.

To facilitate the presentation we will assume that the dissociate sets contain no elements of order 2. Our theorems remain true without this assumption. The technical details with respect to removing this assumption are given at the end of Section 1. Before proceeding to the proofs of these results we establish a preliminary proposition.

Notation. For positive integers $d$ and $N$ and characters $\chi_{1}, \ldots, \chi_{d} \in \Gamma$ let

$$
\begin{aligned}
A_{\infty}\left(N, \chi_{1}, \ldots, \chi_{d}\right) & =\left\{\prod_{j=1}^{d} \chi_{j}^{n_{j}}: \sup _{1 \leq j \leq d}\left|n_{j}\right| \leq N\right\} \\
& =\prod_{j=1}^{d} A_{\infty}\left(N, \chi_{j}\right) .
\end{aligned}
$$


Proposition 1.1. For each positive integer $n$ there are constants $0<\varepsilon(n)<1$ and $C(n)$ so that if $E$ contains no parallelepipeds of dimension $n$ and $\alpha \in \Gamma$ then

$$
\left|E \cap \alpha A_{\infty}\left(N, \chi_{1}, \ldots, \chi_{d}\right)\right| \leq C(n)(2 N+1)^{d \varepsilon(n)}
$$

for every $d, N \in \mathbb{N}$ and $\alpha, \chi_{1}, \ldots, \chi_{d} \in \Gamma$.

REMARK. Related results may be found in [13, Ch. 6].

Proof. This result is stated in [9, Corollary 2.6], but the proof given there only shows that there are constants $C_{1}(n)$ and $0<\varepsilon_{1}(n)<1$ so that

$$
\left|E \cap \alpha A_{\infty}\left(N, \chi_{1}, \ldots, \chi_{d}\right)\right| \leq C_{1}(n) 2^{d \varepsilon_{1}(n)}(2 N+1)^{d \varepsilon_{1}(n)}
$$

for all $d, N \in \mathbb{N}$ and $\alpha, \chi_{1}, \ldots, \chi_{d} \in \Gamma$.

We will prove that

$$
\left|E \cap \alpha A_{\infty}\left(N, \chi_{1}, \ldots, \chi_{d}\right)\right| \leq C_{2}(n)(2 N+1)^{d \varepsilon_{2}(n)+3}
$$

for all $d, N \in \mathbb{N}$ and $\alpha, \chi_{1}, \ldots, \chi_{d} \in \Gamma$.

Once this has been established then by choosing $\varepsilon(n)$ with $\max \left(\varepsilon_{1}(n), \varepsilon_{2}(n)\right)<$ $\varepsilon(n)<1, d_{1}=3\left(\varepsilon(n)-\varepsilon_{2}(n)\right)^{-1}$ and $C(n)=\max \left(C_{1}(n) 2^{d_{1} \varepsilon_{1}(n)}, C_{2}(n)\right)$, we obtain the conclusion of the proposition by using (1) if $d \leq d_{1}$ and (2) if $d>d_{1}$.

To prove (2) we use the method of proof of [14, Theorem 1] and proceed inductively on $n$. Since $E$ contains no parallelepipeds of dimension $n$ if and only if $E \alpha^{-1}$ contains no parallelepipeds of dimension $n$ we may assume without loss of generality that $\alpha=1$. Suppose $n=2$. Let $E_{1}=E \cap A_{\infty}\left(N, \chi_{1}, \ldots, \chi_{d}\right)$. Since $E$ contains no parallelepipeds of dimension two, $\omega_{1} \omega_{2}^{-1} \neq \omega_{3} \omega_{4}^{-1}$ if $\omega_{1}, \omega_{2}, \omega_{3}, \omega_{4}$ are four distinct elements of $E$. Thus

But

$$
\left|E_{1} \cdot E_{1}^{-1}\right| \geq \frac{\left|E_{1}\right|\left(\left|E_{1}\right|-1\right)}{3} .
$$

$$
\left|A_{\infty}\left(N, \chi_{1}, \ldots, \chi_{d}\right) . A_{\infty}\left(N, \chi_{1}, \ldots, \chi_{d}\right)^{-1}\right| \leq(4 N+1)^{d} .
$$

Combining these facts we see that $\left|E_{1}\right| \leq 3(4 N+1)^{d / 2}$, so if we let $C(2)=3$ and $\varepsilon(2)=\frac{1}{2} \log _{2 N+1}(4 N+1)<1$ we obtain $\left|E_{1}\right| \leq C(2)(2 N+1)^{d e(2)}$.

Now assume inductively that

$$
\left|E \cap A_{\infty}\left(N, \chi_{1}, \ldots, \chi_{d}\right)\right| \leq C(n)(2 N+1)^{d \varepsilon(n)+3-3^{3-n}}
$$

if $E$ is any set which contains no parallelepipeds of dimension $n, n \geq 2$.

Let $E$ be a set which contains no parellelepipeds of dimension $n+1$. For $j$ the least integer satisfying

$$
j \geq d\left(\frac{1-\varepsilon(n)}{3}\right)-1+3^{2-n}
$$

let $I_{j}=\prod_{i=1}^{j} A_{\infty}\left(N, \chi_{i}\right)$. (Note that $j \geq 0$. If $j=0$ let $I_{j}=\{1\}$.) Then $\left|I_{j} I_{j}^{-1}\right| \leq(4 N+1)^{j}$ so $I_{j} I_{j}^{-1} \backslash\{1\}=\left\{\beta_{1}, \ldots, \beta_{k}\right\}$ with $k \leq(2 N+1)^{2 j}$. Let $\pi_{1}$ be a maximal collection of two element sets $P_{i}=\left\{\chi_{i}, \psi_{i}\right\}$ with $\chi_{i}, \psi_{i} \in E_{1}, \chi_{i} \psi_{i}^{-1}=\beta_{1}$ and satisfying $P_{i} \cap P_{j}$ is empty if $i \neq j$. Inductively construct $\pi_{l}$ a maximal collection of two element sets $\{\chi, \psi\}$ with 
$\chi \psi^{-1}=\beta_{l}$,

$$
\chi, \psi \in E_{1} \backslash \bigcup_{i=1}^{l-1}\left\{\alpha: \alpha \in P, P \in \pi_{i}\right\}
$$

and satisfying the disjointness condition.

If $\pi_{l}=\left\{\left\{\chi_{i}, \psi_{i}\right\}: i \in J_{l}\right\}$ then $\left\{\chi_{i}\right\}_{i \in J_{l}}$ cannot contain any parallelepipeds of dimension $n$, for otherwise $\left\{\chi_{i}, \psi_{i}: i \in J_{l}\right\} \subseteq E$ would contain a parallelepiped of dimension $n+1$. By the induction assumption

$$
\left|\left\{\psi_{i}: i \in J_{l}\right\}\right|=\left|\left\{\chi_{i}: i \in J_{l}\right\}\right| \leq C(n)(2 N+1)^{d \varepsilon(n)+3-3^{3-n}} .
$$

Observe that if $\chi \in \Gamma$ is chosen so that

then

$$
\chi I_{j} \subseteq A_{\infty}\left(N, \chi_{1}, \ldots, \chi_{d}\right)
$$

$$
\bigcup_{i=1}^{k}\left\{\alpha: \alpha \in P, P \in \pi_{i}\right\}
$$

contains all the elements of $\chi I_{j} \cap E$ except for possibly one point. Since

and

$$
\prod_{i=j+1}^{d} A_{\infty}\left(A, \chi_{i}\right) . I_{j}=A_{\infty}\left(N, \chi_{1}, \ldots, \chi_{d}\right)
$$

it follows that

$$
\left|\prod_{i=j+1}^{d} A_{\infty}\left(N, \chi_{i}\right)\right| \leq(2 N+1)^{d-j}
$$

$$
\left|E \cap A_{\infty}\left(N, \chi_{1}, \ldots, \chi_{d}\right)\right| \leq 2 C(n)(2 N+1)^{d \varepsilon(n)+3-3^{3-n}+2 j}+(2 N+1)^{d-j} .
$$

The choice of $j$ (even in the case $j=0$ ) ensures that

$$
\left|E \cap A_{\infty}\left(N, \chi_{1}, \ldots, \chi_{d}\right)\right| \leq 4 C(n)(2 N+1)^{d((2+E(n)) / 3)+3-3^{2-n}} .
$$

Setting $\varepsilon(n+1)=(2+\varepsilon(n)) / 3<1$ and $C(n+1)=4 C(n)$ we complete the induction step.

Proof of Theorem $A$. Suppose $E_{1}$ contains no parallelepipeds of dimension $n$ and $E \supseteq E_{1}$ is not a Rajchman set. We will show that $E \backslash E_{1}$ cannot be a Rajchman set. By Host and Parreau's characterization of Rajchman sets (Theorem I) we may asume (without loss of generality) that $E$ contains $\Omega(\theta)$ for some infinite dissociate set $\theta=\left\{\theta_{j}\right\}_{j \geq 1} \subseteq \Gamma$.

Observe that $\Omega\left(\left\{\theta_{1}, \ldots, \theta_{k}\right\}\right)=A_{\infty}\left(1, \theta_{1}, \ldots, \theta_{k}\right)$, thus by Proposition 1.1 there are constants $C(n)$ and $0<\varepsilon(n)<1$ so that

$$
\left|E_{1} \cap \Omega\left(\left\{\theta_{1}, \ldots, \theta_{k}\right\}\right)\right| \leq C(n) 3^{k \varepsilon(n)} .
$$

In particular, if $s(0)$ is chosen sufficiently large we can find $\omega_{0} \in \Omega\left(\left\{\theta_{1}, \ldots, \theta_{s(0)}\right\}\right) \backslash E_{1}$. We proceed inductively to select $s(k)$ and a dissociate set

with

$$
\omega_{0}, \omega_{1}, \ldots, \omega_{k} \in \Omega\left(\left\{\theta_{1}, \ldots, \theta_{s(k)}\right\}\right)
$$

$$
\omega_{0} \Omega\left(\left\{\omega_{1}, \ldots, \omega_{k}\right\}\right) \subseteq E \backslash E_{1} .
$$


Now choose $s(k+1)$ satisfying

$$
\frac{3^{s(k+1)-s(k)}-1}{2}>C(n) 3^{s(k+1) e(n)}
$$

For each $\beta \neq 1$ and $\beta \in \Omega\left(\left\{\theta_{s(k)+1}, \ldots, \theta_{s(k+1)}\right\}\right)$ let

$$
A_{\beta}=\omega_{0} \Omega\left(\left\{\omega_{1}, \ldots, \omega_{k}, \beta\right\}\right) \subset \Omega\left(\left\{\theta_{1}, \ldots, \theta_{s(k+1)}\right\}\right) .
$$

Clearly $\left\{\omega_{0}, \omega_{1}, \ldots, \omega_{k}, \beta\right\}$ form a dissociate set. Moreover,

$$
A_{\beta} \cap A_{\gamma}= \begin{cases}A_{\beta}=A_{\beta^{-1}} & \text { if } \gamma=\beta \text { or } \beta^{-1}, \\ \omega_{0} \Omega\left(\left\{\omega_{1}, \ldots, \omega_{k}\right\}\right) & \text { otherwise. }\end{cases}
$$

Hence if $E_{1} \cap A_{\beta}$ is non-empty for every $\beta \in \Omega\left(\left\{\theta_{s(k)+1}, \ldots, \theta_{s(k+1)}\right\}\right) \backslash\{1\}$ we would have

$$
\begin{aligned}
\left|E_{1} \cap \Omega\left(\left\{\theta_{1}, \ldots, \theta_{s(k+1)}\right\}\right)\right| & \geq \frac{1}{2}\left(\left|\Omega\left(\left\{\theta_{s(k)+1}, \ldots, \theta_{s(k+1)}\right\}\right)\right|-1\right) \\
& =\frac{3^{s(k+1)-s(k)}-1}{2}>C(n) 3^{s(k+1) \varepsilon(n)}
\end{aligned}
$$

But this contradicts (3). Thus $A_{\beta} \subseteq E \backslash E_{1}$ for some such $\beta$. Now set $\omega_{k+1}=\beta$. This inductive construction shows that $E \backslash E_{1}$ contains $\omega_{0} \Omega\left(\theta^{\prime}\right)$ for an infinite dissociate set $\theta^{\prime}$.

Definitions. (1) Given $0<p<\infty$ we say $E \subseteq \Gamma$ is a $\Lambda(p)$ set if for some $0<r<p$ there exists a constant $C$ such that $\|f\|_{p} \leq C\|f\|_{r}$ whenever supp $\hat{f}$ is a finite subset of $E$.

(2) Given $1 \leq p<2$ call $E$ a $p$-Sidon set if there is a constant $C$ so that $\|\hat{f}\|_{p} \leq C\|f\|_{\infty}$ whenever supp $\hat{f}$ is a finite subset of $E$. A 1-Sidon set is usually called a Sidon set.

COROLlARY 1.2. If $E$ is a $\Lambda(p)$ set for some $p>0$ or a $p$-Sidon set for $1 \leq p<2$, then the union of $E$ with any Rajchman set is another Rajchman set.

Proof. It is essentially shown in [12, Lemma 1] that $p$-Sidon sets cannot contain parallelepipeds of arbitrarily large dimension. This fact for $\Lambda(p)$ sets, $p>0$, is proved in [9, Theorem 1.2]. (Earlier partial results can be found in [5] and [14].)

Remark. For another example of a class of thin sets which do not contain parallelepipeds of arbitrarily large dimension see [5]. and 1.4.

Proof of Theorem B. Actually we will prove two stronger results, Theorems 1.3

THEOREM 1.3. The union of a strong set of continuity and a set which does not contain parallelepipeds of arbitrarily large dimension is a strong set of continuity.

Proof. The arguments are very similar to those of Theorem A. We suppose $E_{1}$ contains no parallelepipeds of dimension $n$ and $E \supseteq E_{1}$ is not a strong set of continuity. Fix a positive integer $m$. Let $M=s(m)$ where the function $s$ is as in the proof of Theorem A. Without loss of generality assume $E$ contains $\Omega\left(\left\{\theta_{1}, \ldots, \theta_{M}\right\}\right)$ for $\left\{\theta_{1}, \ldots, \theta_{M}\right\}$ a dissociate subset of $\Gamma$. The proof of Theorem $A$ shows that we may select a dissociate subset $\omega_{0}, \omega_{1}, \ldots, \omega_{m} \in \Omega\left(\left\{\theta_{1}, \ldots, \theta_{M}\right\}\right)$ with $\omega_{0} \Omega\left(\left\{\omega_{1}, \ldots, \omega_{m}\right\}\right) \subseteq E \backslash E_{1}$. As $m$ was arbitrary this proves that $E \backslash E_{1}$ is not a strong set of continuity. 
THEOREM 1.4. The union of a proportional set of continuity and a set which does not contain parallelepipeds of arbitrarily large dimension is a proportional set of continuity.

For the proof of Theorem 1.4 we need the following lemma which is similar in spirit to Proposition 1.1.

LEMma 1.5. There are constants $C(n)$ and $0<\varepsilon(n)<1$ so that if $E \subseteq \Gamma$ contains no parallelepipeds of dimension $n$ then, for any positive integers $m$ and $N, \alpha \in \Gamma$ and dissociate set $\left\{\theta_{1}, \ldots, \theta_{N}\right\} \subseteq \Gamma$,

$$
\left|E \cap \alpha \Omega_{m}\left(\left\{\theta_{1}, \ldots, \theta_{N}\right\}\right)\right| \leq C(n) \sum_{k=0}^{m}\left(\begin{array}{l}
N \\
k
\end{array}\right) 2^{k \varepsilon(n)} .
$$

Proof. Without loss of generality assume $\alpha=1$. For $k=1,2, \ldots$, let

$$
M_{k}\left(\left\{\theta_{1}, \ldots, \theta_{N}\right\}\right) \equiv\left\{\prod_{i=1}^{N} \theta_{i}^{\varepsilon_{i}}: \varepsilon_{i}=0, \pm 1, \sum_{i=1}^{N}\left|\varepsilon_{i}\right|=k\right\} .
$$

For $\sigma=\left\{i_{1}, \ldots, i_{k}\right\} \subset\{1, \ldots, N\}$ consider the parallelepiped of dimension $k$

$$
P_{\sigma}=\prod_{j=1}^{k}\left\{\theta_{i_{i}}, \theta_{i_{j}}^{-1}\right\} .
$$

It is clear that $M_{k}\left(\left\{\theta_{1}, \ldots, \theta_{N}\right\}\right)=\bigcup P_{o}$ where the union is taken over all subsets $\sigma$ of $\left\{\theta_{1}, \ldots, \theta_{N}\right\}$ of cardinality $k$. It is known [9, Proposition 2.2] that there are constants $C(n) \geq 1$ and $0<\varepsilon(n)<1$ such that

$$
\left|E \cap P_{\sigma}\right| \leq C(n) 2^{k \in(n)} .
$$

Since $\Omega_{m}\left(\left\{\theta_{1}, \ldots, \theta_{N}\right\}\right)=\{1\} \cup \bigcup_{k=1}^{m} M_{k}\left(\left\{\theta_{1}, \ldots, \theta_{N}\right\}\right)$ we have that

$$
\begin{aligned}
\left|E \cap \Omega_{m}\left(\left\{\theta_{1}, \ldots, \theta_{N}\right\}\right)\right| & \leq 1+\sum_{k=1}^{m}\left|E \cap M_{k}\left(\left\{\theta_{1}, \ldots, \theta_{N}\right\}\right)\right| \\
& \leq \sum_{k=0}^{m} C(n) 2^{k \varepsilon(n)}\left(\begin{array}{l}
N \\
k
\end{array}\right) .
\end{aligned}
$$

COROLlarY 1.6. Suppose $E \subseteq \Gamma$ does not contain parallelepipeds of arbitrarily large dimension. Then for every $\delta>0$ there exists an integer $m_{0}$ such that for all $m \geq m_{0}$, any infinite dissociate set $\theta=\left\{\theta_{i}\right\} \subseteq \Gamma$, and $\alpha \in \Gamma$

$$
\limsup _{N \rightarrow \infty} \frac{\left|E \cap \alpha \Omega_{m}\left(\left\{\theta_{1}, \ldots, \theta_{N}\right\}\right)\right|}{\left|\alpha \Omega_{m}\left(\left\{\theta_{1}, \ldots, \theta_{N}\right\}\right)\right|} \leq \delta .
$$

In particular, $E$ is a proportional set of continuity.

Proof. This follows easily from the lemma since

$$
\left|\alpha \Omega_{m}\left(\left\{\theta_{1}, \ldots, \theta_{N}\right\}\right)\right|=\sum_{k=0}^{m} 2^{k}\left(\begin{array}{l}
N \\
k
\end{array}\right) .
$$

Proof of Theorem 1.4. Suppose $E_{1} \subset \Gamma$ contains no parallelepipeds of dimension $n$. Suppose $E_{2}$ is a proportional set of continuity with proportionality constant $C<1$. 
Choose $C_{1}$ with $C<C_{1}<1$. Choose $m_{0} \geq n_{0}\left(n_{0}\right.$ as in the definition of a proportional set of continuity) so large that if $m \geq m_{0}$ then

$$
\underset{N \rightarrow \infty}{\limsup } \frac{\left|E_{1} \cap \alpha \Omega_{m}\left(\left\{\theta_{1}, \ldots, \theta_{N}\right\}\right)\right|}{\left|\alpha \Omega_{m}\left(\left\{\theta_{1}, \ldots, \theta_{N}\right\}\right)\right|} \leq C_{1}-C
$$

for any $\theta=\left\{\theta_{i}\right\}_{i \geq 1}$ an infinite dissociate set and $\alpha \in \Gamma$. Thus if $m \geq m_{0}$,

$$
\begin{aligned}
& \liminf _{N \rightarrow \infty} \frac{\left|\left(E_{1} \cup E_{2}\right) \cap \alpha \Omega_{m}\left(\left\{\theta_{1}, \ldots, \theta_{N}\right\}\right)\right|}{\left|\alpha \Omega_{m}\left(\left\{\theta_{1}, \ldots, \theta_{N}\right\}\right)\right|} \\
& \quad \leq \limsup _{N \rightarrow \infty} \frac{\left|E_{1} \cap \alpha \Omega_{m}\left(\left\{\theta_{1}, \ldots, \theta_{N}\right\}\right)\right|}{\left|\alpha \Omega_{m}\left(\left\{\theta_{1}, \ldots, \theta_{N}\right\}\right)\right|}+C \leq C_{1}<1 .
\end{aligned}
$$

Thus $E_{1} \cup E_{2}$ is a proportional set of continuity.

COROLlary 1.7. The union of a proportional set of continuity and either a $\Lambda(p)$ set for some $p>0$ or a $p$-Sidon set for $1 \leq p<2$ is a proportional set of continuity.

REMARK. For Theorems A and 1.3 it is not difficult to handle the case when there are elements of order 2. Consider the situation in Theorem A. Suppose $E \supseteq \Omega(\theta)$ for some infinite dissociate set $\theta$. Let $\theta=\theta_{1} \cup \theta_{2}$ where $\theta_{1}$ consists of those elements of $\theta$ which are of order 2 and $\theta_{2}$ consists of those which are not of order 2. At least one of $\theta_{1}$ or $\theta_{2}$ is an infinite set. If $\theta_{2}$ is an infinite set then since $E \supset \Omega\left(\theta_{2}\right)$, from the non-order 2 version of Theorem A we conclude that $E \backslash E_{1}$ is not a Rajchman set, which is a contradiction.

If $\theta_{1}$ is an infinite set we first use arguments similar to those used in Proposition 1.1 to prove

Proposition 1.1'. For each positive integer $n$ there are constants $0<\varepsilon(n)<1$ and $C(n)$ such that if $E$ contains no parallelepipeds of dimension $n$ and $\alpha \in \Gamma$ then

$$
\left|E \cap \alpha A_{\infty}\left(N, \chi_{1}, \ldots, \chi_{d}\right)\right| \leq C(n)(N+1)^{d \varepsilon(n)}
$$

for every $d, N \in \mathbb{N}$ and $\alpha, \chi_{1}, \ldots, \chi_{d} \in \Gamma$ with $\chi_{1}, \ldots, \chi_{d}$ each having order 2.

The main point here is that, in this case, $\left|A_{\infty}\left(N, \chi_{1}, \ldots, \chi_{d}\right)\right| \leq(N+1)^{d}$. Theorem A can then be proved by restricting our attention to $\theta_{1}=\left\{\chi_{i}\right\}$ and using the fact that

$$
\left|\Omega\left(\left\{\chi_{1}, \ldots, \chi_{d}\right\}\right)\right|=2^{d} \text {. }
$$

Theorem 1.3 can be proved similarly.

To prove Theorem 1.4 we cannot, unfortunately, restrict our attention to one of $\theta_{1}$ or $\theta_{2}$. Instead we use the fact that Corollary 1.6 remains true if the dissociate set contains elements of order 2 . The ideas are again combinatorial, but are more involved. An outline of the proof is given below.

Proof of Corollary 1.6 when the dissociate set may contain elements of order 2. First we remark that without loss of generality we may assume $\alpha=1$. Let $\theta=\left\{\theta_{i}\right\}$ be an infinite dissociate set. For each positive integer $N$, let $\left\{\chi_{i}\right\}_{i=1}^{(N)} \subseteq\left\{\theta_{i}\right\}_{i=1}^{N}$ consist of those elements of order 2 , and let $\left\{\psi_{i}\right\}_{i=1}^{L(N)} \subseteq\left\{\theta_{i}\right\}_{i=1}^{N}$ be the remaining terms. If $\sup _{N} J(N)=J_{0}<\infty$ then, for any positive integer $m$,

$$
\Omega_{m}\left(\left\{\theta_{1}, \ldots, \theta_{N}\right\}\right) \subseteq \Omega_{m}\left(\left\{\chi_{1}, \ldots, \chi_{J(N)}\right\}\right) . \Omega_{m}\left(\left\{\psi_{1}, \ldots, \psi_{L(N)}\right\}\right) .
$$


Thus

$$
\frac{\left|E \cap \Omega_{m}\left(\left\{\theta_{1}, \ldots, \theta_{N}\right\}\right)\right|}{\left|\Omega_{m}\left(\left\{\theta_{1}, \ldots, \theta_{N}\right\}\right)\right|} \leq 2^{J_{0}} \sup _{\alpha \in \Omega\left(\left\{\chi_{1}, \ldots, \chi_{J_{0}}\right\}\right)} \frac{\left|E \cap \alpha \cdot \Omega_{m}\left(\left\{\psi_{1}, \ldots, \psi_{L(N)}\right\}\right)\right|}{\left|\Omega_{m}\left(\left\{\psi_{1}, \ldots, \psi_{L(N)}\right\}\right)\right|}
$$

and hence this version of Corollary 1.6 follows from the case where the order is not equal to 2 .

Thus we may assume $\lim _{N} J(N)=\sup _{N} J(N)=\infty$. As

$$
\begin{aligned}
& \Omega_{m}\left(\left\{\theta_{1}, \ldots, \theta_{N}\right\}\right)=\Omega_{m}\left(\left\{\chi_{2}, \ldots, \chi_{J(N)}, \psi_{1}, \ldots, \psi_{L(N)}\right\}\right) \\
& \cup \chi_{1} \Omega_{m-1}\left(\left\{\chi_{2}, \ldots, \chi_{J(N)}, \psi_{1}, \ldots, \psi_{L(N)}\right\}\right)
\end{aligned}
$$

we may without loss of generality assume $J(N)$ is even. We will use the same notation as in the proof of Lemma 1.5. We will write $J(N)$ for $J(N) / 2$ as it arises frequently.

Observe that

$$
M_{n}\left(\left\{\theta_{1}, \ldots, \theta_{N}\right\}\right)=\bigcup_{k} M_{k}\left(\left\{\chi_{1}, \ldots, \chi_{J(N)}\right\}\right) M_{n-k}\left(\left\{\psi_{1}, \ldots, \psi_{L(N)}\right\}\right),
$$

where the union is over $k \geq 0$ with $n-L(N) \leq k \leq \min \left(n, J(N)\right.$ ) (let $\left.M_{0}=\{1\}\right)$. Since we are interested in the behaviour as $N \rightarrow \infty$ we may assume $k \leq n \leq \tilde{J}(N)$. As in Lemma 1.5 $M_{n-k}$ is a union of $\left(\begin{array}{l}L(N) \\ n-k\end{array}\right)$ parallelepipeds $P_{\sigma}$ of dimension $n-k$.

For $\pi=\left\{i_{1}, \ldots, i_{k}\right\} \subseteq\{1, \ldots, \tilde{J}(N)\}$ let

$$
Q_{\pi}=\prod_{j=1}^{k}\left\{\chi_{2 i_{j}-1}, \chi_{2 i j}\right\}
$$

For those positive integers $j$ such that $k-2 j \geq 0$ let

where

$$
\tau(j)=\left\{i_{1}, \ldots, i_{j}\right\} \cup\left\{l_{1}, \ldots, l_{k-2, j}\right\}
$$

$$
\begin{gathered}
\left\{i_{1}, \ldots, i_{j}\right\} \subset\{1, \ldots, \tilde{J}(N)\}, \\
\left\{l_{1}, \ldots, l_{k-2 j}\right\} \subset\{1, \ldots, \tilde{J}(N)\} \backslash\left\{i_{1}, \ldots, i_{j}\right\} .
\end{gathered}
$$

(If $k-2 j=0$ let $\tau(j)=\left\{i_{1}, \ldots, i_{j}\right\}$ ). Let

$$
R_{\tau(j)}=\prod_{s=1}^{j} \chi_{2 i_{s}-1} \chi_{2 i_{s}} \prod_{t=1}^{k-2 j}\left\{\chi_{2 i_{t}-1}, \chi_{2 l_{t}}\right\}
$$

(with the usual convention that the empty product is 1). Note that there are $\left(\begin{array}{c}\tilde{J}(N) \\ k\end{array}\right)$ choices for $\pi$ and

choices for $\tau(j)$.

$$
\sum_{j=1}^{[k / 2]}\left(\begin{array}{c}
\tilde{J}(N) \\
j
\end{array}\right)\left(\begin{array}{c}
\tilde{J}(N)-j \\
k-2 j
\end{array}\right)
$$


Since for $k \leq \tilde{J}(N)$

we see that

$$
M_{k}\left(\left\{\chi_{1}, \ldots, \chi_{J(N)}\right\}\right)=\bigcup_{\pi} Q_{\pi} \cup \bigcup_{\tau(j)} R_{\tau(j)}
$$

$$
\left|M_{k}\left(\left\{\chi_{1}, \ldots, \chi_{J(N)}\right\}\right)\right|=\left(\begin{array}{c}
J(N) \\
k
\end{array}\right)=\left(\begin{array}{c}
\tilde{J}(N) \\
k
\end{array}\right) 2^{k}+\sum_{j=1}^{[k / 2]}\left(\begin{array}{c}
\tilde{J}(N) \\
j
\end{array}\right)\left(\begin{array}{c}
\tilde{J}(N)-j \\
k-2 j
\end{array}\right) 2^{k-2 j}
$$

Since $P_{\sigma} Q_{\pi}$ and $P_{\sigma} R_{\tau(j)}$ are parallelepipeds of dimension at most $n$,

$$
\left|E \cap P_{\sigma} Q_{\pi}\right| \leq C 2^{n \varepsilon} \text { and }\left|E \cap P_{\sigma} R_{\tau(j)}\right| \leq C 2^{n E}
$$

for some constant $C$ and $0<\varepsilon<1$ [9, Proposition 2.2]. Thus, since

we have

$$
M_{n}\left(\left\{\theta_{1}, \ldots, \theta_{N}\right\}\right)=\bigcup_{\sigma, \pi} P_{\sigma} Q_{\pi} \cup \bigcup_{\sigma, \tau(j)} P_{\sigma} R_{\tau(j)}
$$

$$
\left|E \cap M_{n}\left(\left\{\theta_{1}, \ldots, \theta_{N}\right\}\right)\right| \leq C 2^{n \varepsilon} \sum_{k}^{\prime}\left(\begin{array}{c}
L(N) \\
n-k
\end{array}\right)\left[\left(\begin{array}{c}
\tilde{J}(N) \\
k
\end{array}\right)+\sum_{j=1}^{\mid k / 2\}}\left(\begin{array}{c}
\tilde{J}(N) \\
j
\end{array}\right)\left(\begin{array}{c}
\tilde{J}(N)-j \\
k-2 j
\end{array}\right)\right],
$$

where $\sum_{k}^{\prime}$ denotes the sum over those $k$ such that

$$
\max (0, n-L(N)) \leq k \leq n .
$$

Furthermore,

$$
\left|M_{n}\left(\left\{\theta_{1}, \ldots, \theta_{N}\right\}\right)\right|=\sum_{k}^{\prime}\left(\begin{array}{c}
J(N) \\
k
\end{array}\right)\left(\begin{array}{c}
L(N) \\
n-k
\end{array}\right) 2^{n-k}
$$

By combining (4), (5), (6) and the fact that

$$
\underset{N}{\limsup } \frac{\left|E \cap \Omega_{m}\left(\left\{\theta_{1}, \ldots, \theta_{N}\right\}\right)\right|}{\left|\Omega_{m}\left(\left\{\theta_{1}, \ldots, \theta_{N}\right\}\right)\right|} \leq \limsup _{N} \frac{1+\sum_{n=1}^{m}\left|E \cap M_{n}\left(\left\{\theta_{1}, \ldots, \theta_{N}\right\}\right)\right|}{1+\sum_{n=1}^{m}\left|M_{n}\left(\left\{\theta_{1}, \ldots, \theta_{N}\right\}\right)\right|},
$$

tedious calculations now establish the desired result. An interested reader can find the remaining details in the Appendix.

2. Random thin sets. It is unknown if there is an arithmetic property which characterizes $\Lambda(p)$ sets. In [9, Section 4] probabilistic methods of Erdös \& Rényi [4] (see also $[7,4.5-4.8])$ are used to show that for every $p>8 / 3$ there is a set $E(p) \subset \mathbb{Z}$ which does not contain parallelepipeds of arbitrarily large dimension and yet is not a $\Lambda(p)$ set. We use similar methods here to extend this result to any $p>2$. Actually, the sets which we will construct have an even stronger arithmetic property.

Definition 2.1. A subset $E$ of $\mathbb{Z}$ is said to contain a square of size $N^{2}$ if there are $N$-element sets $A, B \subset \mathbb{Z}$, with $A+B \subset E$ and $|A+B|=N^{2}$. (As is customary we use addition to denote the group operation on $\mathbb{Z}$.)

Observe that if $E$ contains a parallelepiped of dimension $2 N$, then $E$ contains a square of size $2^{2 N}$. 
It is well known that Sidon sets cannot contain arbitrarily large squares [13, Theorem 1.4]. A consequence of the work of Blei [1] is that for each $1 \leq p<2$ there are sets which do not contain arbitrarily large squares and yet are not $p$-Sidon. The random construction we give below yields this fact as well.

THEOREM 2.2. For every $0<\alpha<1$ there is a set $E(\alpha) \subset \mathbb{Z}$ which does not contain arbitrarily large squares and yet $|E(\alpha) \cap[1, N]| \geq O\left(N^{1-\alpha}\right)$.

Proof. Let $0<\alpha<1$ and let $\left\{\xi_{n}\right\}_{n=2}^{\infty}$ be a sequence of independent random variables such that $P\left(\xi_{n}=1\right)=p_{n}=1 / n^{\alpha}$ and $P\left(\xi_{n}=0\right)=1-p_{n}$. Let $\left\{v_{k}\right\}$ denote the values of $n$ (in increasing order) with $\xi_{n}=1$. Thus $p_{n}$ is the probability that $n$ is contained in $\left\{v_{k}\right\}$.

Choose an integer $N>2 / \alpha$. Observe that if $\left\{v_{k}\right\}$ contains a square of size $N^{2}$ then $\left\{v_{k}\right\}$ contains a square of size $N^{2}$ of the form $\left\{a_{i}+b_{j}: i, j=1, \ldots, N\right\}$ with $a_{i}, b_{j} \geq 1$.

Since the numbers $\left\{a_{i}+b_{j}\right\}_{i, j=1}^{N}$ are distinct

$$
P\left(\left\{a_{i}+b_{j}\right\}_{i, j=1}^{N} \subset\left\{v_{k}\right\}\right)=\prod_{i, j=1}^{N} \frac{1}{\left(a_{i}+b_{j}\right)} \alpha .
$$

By using the inequality $a+b \geq a^{1 / 2} b^{1 / 2}$ for $a, b \geq 0$ we see that

$$
P\left(\left\{a_{i}+b_{j}\right\}_{i, j=1}^{N} \subset\left\{v_{k}\right\}\right) \leq \frac{1}{a_{1}^{N \alpha / 2} \ldots a_{N}^{N \alpha / 2} b_{1}^{N \alpha / 2} \ldots b_{N}^{N \alpha / 2}} .
$$

Thus if $\sum_{N}^{\prime}$ denotes the sum over those positive integers $a_{1}, \ldots, a_{N}, b_{1}, \ldots, b_{N}$ such that $\left|\left\{a_{i}+b_{j}\right\}_{i, j=1}^{N}\right|=N^{2}$ then

$$
\sum_{N}^{\prime} P\left(\left\{a_{i}+b_{j}\right\}_{i, j=1}^{N} \subset\left\{v_{k}\right\}\right) \leq\left(\sum_{a \in \mathbf{Z}^{+}} \frac{1}{a^{N \alpha / 2}}\right)^{N}\left(\sum_{b \in \mathbf{Z}^{+}} \frac{1}{b^{N \alpha / 2}}\right)^{N}<\infty
$$

since $N>2 / \alpha$.

An application of the Borel-Cantelli lemma shows that $\left\{v_{k}\right\}$ contains only finitely many squares of size $N^{2}$ a.s.

Since

and

$$
E\left(\xi_{n}\right)>0, \quad \lim _{n \rightarrow \infty} E\left(\sum_{i \leq n} \xi_{i}\right)=\infty
$$

$$
\sum_{n} \frac{\operatorname{Var}\left(\xi_{n}\right)}{E\left(\sum_{i \leq n} \xi_{i}\right)^{2}}=\sum_{n} \frac{p_{n}\left(1-p_{n}\right)}{\left(p_{1}+\ldots+p_{n}\right)^{2}} \leq \sum_{n} \frac{1}{n^{\alpha} n^{2(1-\alpha)}}<\infty,
$$

by a variant of the strong law of large numbers (cf. [8, p. 140])

$$
\lim _{n \rightarrow \infty} \frac{E\left(\sum_{i \leq n} \xi_{i}\right)}{\sum_{i \leq n} \xi_{i}}=1 \text { a.s. }
$$


Thus

$$
\lim _{k \rightarrow \infty} \frac{\sum_{i \leq v_{k}} p_{i}}{k}=\lim _{k \rightarrow \infty} \frac{v_{k}^{1-\alpha}}{(1-\alpha) k}=1 \quad \text { a.s. }
$$

and so there is a $c>0$ such that for all $N$ sufficiently large,

$$
\left|\left\{v_{k}\right\} \cap[1, N]\right| \geqslant c N^{1-\alpha} \text { a.s. }
$$

Let $E(\alpha)$ be such a set $\left\{v_{k}\right\}$ with the finitely many squares of size $N^{2}$ deleted. Since $E(\alpha)$ and $\left\{v_{k}\right\}$ have the same asymptotic density the theorem is proved.

COROLlaRY 2.3. For every $p>2$ there is a set $E(p)$ which does not contain arbitrarily large squares, and so in particular does not contain parallelepipeds of arbitrarily large dimension, and yet is not a $\Lambda(p)$ set.

Proof. Choose $0<\alpha<1$ with $1-\alpha>2 / p$ and take $E(p)$ equal to the set $E(\alpha)$ as constructed in the theorem. If $E(p)$ was a $\Lambda(p)$ set then by [17, Theorem 3.5]

but this not so.

$$
|E \cap[1, N]| \leq C N^{2 / p} \text {, }
$$

COROLlaRY 2.4. There is a set $E$ which does not contain arbitrarily large squares but is not a $p$-Sidon set for any $1 \leq p<2$.

Proof. It is shown in [3, Corollary 2.6] that if $E$ is a $p$-Sidon set then

$$
|E \cap[1, N]| \leq C(\log N)^{1 / 2 a}
$$

where $a=2 p /(3 p-2)$. Thus $E(\alpha)$ is never $p$-Sidon for any $0<\alpha<1$ and $1 \leq p<2$.

Appendix. Completion of the proof of Corollary 1.6 when the dissociate set may contain elements of order 2. We continue using the notation described in the earlier outline of the proof.

Choose $r>1$ so that $1 / r+\varepsilon<1$. Equation (4) implies that for $k \leq n \leq \tilde{J}(N)$

$$
\left(\begin{array}{c}
J(N) \\
k
\end{array}\right) 2^{n / r} \geq\left(\begin{array}{c}
\tilde{J}(N) \\
k
\end{array}\right) 2^{k}+\sum_{j=1}^{[k /(2 r)]}\left(\begin{array}{c}
\tilde{J}(N) \\
j
\end{array}\right)\left(\begin{array}{c}
\tilde{J}(N)-j \\
k-2 j
\end{array}\right) 2^{k-2 j} 2^{2 j}
$$

(If $k /(2 r)<1$ omit the second term on the right.)

From (5), (6), (8) and the trivial fact that for positive numbers $a_{i}, b_{i}$

$$
\begin{aligned}
& \underset{N}{\lim \sup } \frac{\left|E \cap M_{n}\left(\left\{\theta_{1}, \ldots, \theta_{N}\right\}\right)\right|}{\left|M_{n}\left(\left\{\theta_{1}, \ldots, \theta_{N}\right\}\right)\right|} \\
& \frac{\sum_{i=1}^{M} a_{i}}{\sum_{i=1}^{M} b_{i}} \leq \sum_{i=1}^{M} \frac{a_{i}}{b_{i}} \\
& \leq \underset{N}{\lim \sup } C \frac{2^{n \varepsilon}}{2^{n}} \sum_{k}^{\prime} \frac{\left(\begin{array}{c}
L(N) \\
n-k
\end{array}\right)\left[\left(\begin{array}{c}
\tilde{J}(N) \\
k
\end{array}\right)+\sum_{j=1}^{[k / 2]}\left(\begin{array}{c}
\tilde{J}(N) \\
j
\end{array}\right)\left(\begin{array}{c}
\tilde{J}(N)-j \\
k-2 j
\end{array}\right)\right]}{\left(\begin{array}{c}
L(N) \\
n-k
\end{array}\right)\left(\begin{array}{c}
J(N) \\
k
\end{array}\right) 2^{-k}} \\
& \leq \lim \sup _{N} C \frac{2^{n \varepsilon}}{2^{n}} \sum_{k}^{\prime}\left[2^{n / r}+\sum_{j=[k /(2 r)]+1}^{[k / 2]} \frac{\left(\begin{array}{c}
\tilde{J}(N) \\
j
\end{array}\right)\left(\begin{array}{c}
\tilde{J}(N)-j \\
k-2 j
\end{array}\right) 2^{k}}{\left(\begin{array}{c}
J(N) \\
k
\end{array}\right)}\right] .
\end{aligned}
$$


Observe that for $j=[k /(2 r)]+1, \ldots,[k / 2]$

$$
\left(\begin{array}{c}
\tilde{J}(N) \\
j
\end{array}\right)\left(\begin{array}{c}
\tilde{J}(N)-j \\
k-2 j
\end{array}\right) \leq(\tilde{J}(N))^{k(1-1 /(2 r))}
$$

Also, for $J(N)$ sufficiently large,

$$
\left(\begin{array}{c}
J(N) \\
k
\end{array}\right) \geq \frac{(\tilde{J}(N))^{k}}{k !}
$$

Thus

$$
\begin{aligned}
\limsup _{N} & \frac{\left|E \cap M_{n}\left(\left\{\theta_{1}, \ldots, \theta_{N}\right\}\right)\right|}{\left|M_{n}\left(\left\{\theta_{1}, \ldots, \theta_{N}\right\}\right)\right|} \\
& \leq \limsup _{N} C \frac{2^{n \varepsilon}}{2^{n}}\left[n 2^{n / r}+\sum_{k=1}^{n} \sum_{j=[k /(2 r)]+1}^{[k /(2 r)]} \frac{(\tilde{J}(N))^{k(1-1 /(2 r))} 2^{k} k !}{(\tilde{J}(N))^{k}}\right] \\
& \leq \limsup _{N}\left(C \frac{2^{n(e+1 / r)} n}{2^{n}}+C \frac{2^{n \varepsilon}}{2^{n}} \frac{n^{2} / 2^{n+1} n !}{\tilde{J}(N)^{1 /(2 r)}}\right) \\
& =C 2^{-n(1-\varepsilon-1 / r)} n .
\end{aligned}
$$

Furthermore we note that from (6) again

$$
\underset{N}{\limsup } \frac{\left|M_{n}\left(\left\{\theta_{1}, \ldots, \theta_{N}\right\}\right)\right|}{\left.\mid M_{m}\left\{\theta_{1}, \ldots, \theta_{N}\right\}\right) \mid}=\limsup _{N} \frac{\sum_{k \geq \max (0, n-L(N))}^{n}\left(\begin{array}{c}
J(N) \\
k
\end{array}\right)\left(\begin{array}{c}
L(N) \\
n-k
\end{array}\right) 2^{n-k}}{\sum_{k \geq \max (0, m-L(N))}^{m}\left(\begin{array}{c}
J(N) \\
k
\end{array}\right)\left(\begin{array}{c}
L(N) \\
m-k
\end{array}\right) 2^{m-k}} .
$$

Suppose $n \leq[m / 3]$. If $\limsup _{N} L(N)<\infty$ then

$$
\begin{aligned}
\limsup _{N} \frac{\left|M_{n}\left(\left\{\theta_{1}, \ldots, \theta_{N}\right\}\right)\right|}{\left|M_{m}\left(\left\{\theta_{1}, \ldots, \theta_{N}\right\}\right)\right|} & \leq \limsup _{N} \frac{\max _{0 \leq k \leq n}\left(\begin{array}{c}
J(N) \\
k
\end{array}\right) \sum_{j=0}^{L(N)}\left(\begin{array}{c}
L(N) \\
j
\end{array}\right) 2^{j}}{\left(\begin{array}{c}
J(N) \\
m
\end{array}\right)} \\
& \leq \limsup _{N} \frac{3^{L(N)}\left(\begin{array}{c}
J(N) \\
{[m / 3]}
\end{array}\right)}{\left(\begin{array}{c}
J(N) \\
m
\end{array}\right)}=0 .
\end{aligned}
$$

If $\limsup _{N} L(N)=\infty$ then

$$
\underset{N}{\limsup } \frac{\left|M_{n}\left(\left\{\theta_{1}, \ldots, \theta_{N}\right\}\right)\right|}{\left|M_{m}\left(\left\{\theta_{1}, \ldots, \theta_{N}\right\}\right)\right|} \leq \limsup \sum_{N=0}^{n} \frac{\left(\begin{array}{l}
L(N) \\
n-k
\end{array}\right) 2^{n-k}}{\left(\begin{array}{c}
L(N) \\
m-k
\end{array}\right) 2^{m-k}}
$$


Hence

$$
\begin{aligned}
& \leq \limsup _{N} 2^{-2 m / 3} \frac{m}{3} \max _{0 \leq k \leq n} \frac{\left(\begin{array}{l}
L(N) \\
n-k
\end{array}\right)}{\left(\begin{array}{l}
L(N) \\
m-k
\end{array}\right)} \\
& \leq \limsup 2^{-2 m / 3} \frac{m}{3} \frac{\left(\begin{array}{c}
L(N) \\
{[m / 3]}
\end{array}\right)}{\left(\begin{array}{c}
L(N) \\
m-[m / 3]
\end{array}\right)}=0 .
\end{aligned}
$$

$$
\limsup \frac{1+\sum_{n=1}^{[m / 3]}\left|M_{n}\left(\left\{\theta_{1}, \ldots, \theta_{N}\right\}\right)\right|}{\left|M_{m}\left(\left\{\theta_{1}, \ldots, \theta_{N}\right\}\right)\right|}=0 .
$$

Finally, returning to equation (7) we have

$$
\begin{aligned}
\lim \sup _{N} & \frac{\left|E \cap \Omega_{m}\left(\left\{\theta_{1}, \ldots, \theta_{N}\right\}\right)\right|}{\left|\Omega_{m}\left(\left\{\theta_{1}, \ldots, \theta_{N}\right\}\right)\right|} \\
\quad \leq & \limsup _{N} \frac{1+\sum_{n=1}^{[m / 3]}\left|M_{n}\left(\left\{\theta_{1}, \ldots, \theta_{N}\right\}\right)\right|}{\left|M_{m}\left(\left\{\theta_{1}, \ldots, \theta_{N}\right\}\right)\right|}+\sum_{n=[m / 3]+1}^{m} \limsup _{N} \frac{\left|E \cap M_{n}\left(\left\{\theta_{1}, \ldots, \theta_{N}\right\}\right)\right|}{\left|M_{n}\left(\left\{\theta_{1}, \ldots, \theta_{N}\right\}\right)\right|} \\
& \leq \sum_{n=[m / 3]+1}^{m} C 2^{-n(1-\varepsilon-(1 / r))} n \quad(\text { by }(9) \&(10)) \\
& \leq \frac{C 2^{-(m / 3)(1-\varepsilon-(1 / r))} m}{1-2^{-(1-\varepsilon-(1 / r))}} .
\end{aligned}
$$

As $\varepsilon+(1 / r)<1$ this can be made less than $\delta$ provided $m$ is chosen sufficiently large.

\section{REFERENCES}

1. R. Blei, Fractional Cartesian products of sets, Ann. Inst. Fourier (Grenoble) 29 (1979), 79-105.

2. K. de Leeuw and Y. Katznelson, The two sides of a Fourier-Stieltjes transform and almost idempotent measures, Israel J. Math. 8 (1970), 213-229.

3. R. E. Edwards and K. A. Ross, p-Sidon sets, J. Funct. Anal. 15 (1974), 404-427.

4. P. Erdös and A. Rényi, Additive properties of random sequences of positive integers, Acta Arith. 6 (1960), 83-110.

5. J. Fournier and L. Pigno, Analytic and arithmetic properties of thin sets, Pacific J. Math. 105 (1983), 115-141.

6. P. Gardner and L. Pigno, The two sides of a Fourier-Stieltjes transform, Arch. Math. (Basel) 32 (1979), 75-78.

7. D. Hajela, Construction techniques for some thin sets in duals of compact abelian groups, Ann. Inst. Fourier (Grenoble) 36 (1986), 137-166.

8. H. Halberstam and K. Roth, Sequences, Volume 1 (Clarendon Press, 1966).

9. K. E. Hare, Arithmetic properties of thin sets, Pacific J. Math. 131 (1988), 143-155.

10. B. Host and F. Parreau, Ensembles de Rajchman et ensembles de continuité, C.R. Acad. Sci. Paris Sér. I Math. 288 (1979), 899-902. 
11. B. Host and F. Parreau, Sur les mesures dont la transformée de Fourier Stieltjes ne tend pas vers zéro à l'infini, Colloq. Math. 41 (1979), 285-289.

12. G. W. Johnson and G. S. Woodward, On p-Sidon sets, Indiana Univ. Math. J. 24 (1974), 161-167.

13. J. Lopez and K. Ross, Sidon sets, Lecture notes in Pure and Applied Mathematics 13 (Marcel Dekker, 1975).

14. I. Miheev, Trigonometric series with gaps, Anal. Math. 9 (1983), 43-55.

15. L. Pigno, Fourier transforms which vanish at infinity off certain sets, Glasgow Math. J. 19 (1978), 49-56.

16. A. Rajchman, Une classe de séries trigonométriques qui convergent presque partout vers zéro, Math. Ann. 101 (1929), 686-700.

17. W. Rudin, Trigonometric series with gaps, J. Math. Mech. 9 (1960), 203-227.

Department of Pure Mathematics

UNIVERSITY OF WATERLOO

Waterloo, Ontario N2L 3G1

CANADA 